\title{
Correction: Comprehensive analysis of 204 sporadic hydatidiform moles: revisiting risk factors and their correlations with the molar genotypes
}

Yassemine Khawajkie - Nawel Mechtouf $\cdot$ Ngoc Minh Phuong Nguyen $\cdot$ Kurosh Rahimi $\cdot$ Magali Breguet Jocelyne Arseneau • Brigitte M. Ronnett • Lori Hoffner • Felicia Lazure • Marjolaine Arnaud • Fabrice Peers • Liane Tan - Basam Abu Rafea - Monica Aguinaga - Neil S. Horowitz - Asangla Ao · Seang Lin Tan - Richard Brown • William Buckett $\cdot$ Urvashi Surti $\cdot$ Karine Hovanes $\cdot$ Trilochan Sahoo $\cdot$ Philippe Sauthier $\cdot$ Rima Slim $(\mathbb{D}$

Published online: 12 February 2020

(c) The Author(s), under exclusive licence to United States \& Canadian Academy of Pathology 2020

\section{Correction to: Modern Pathology} https://doi.org/10.1038/s41379-019-0432-4

Author 'Monica Aguinaga' was listed in the original article as 'Monica Aguinuaga'. However, this should be listed as
'Monica Aguinaga'. This has been addressed by means of this correction article as well as an update to the original article. 